\title{
A Scale-free Business Network for Digital Ecosystems
}

\author{
Amir R Razavi, Sotiris K Moschoyiannis, Member, IEEE and Paul J Krause \\ Department of Computing, School of Electronics and Physical Sciences, University of Surrey, \\ Guildford, Surrey, GU2 7XH, UK, \\ e-mail : (a.razavi, s.moschoyiannis, p.krause)@surrey.ac.uk
}

\begin{abstract}
The aim of this paper is to facilitate e-business transactions between Small and Medium Enterprises (SMEs), in a way that respects their local autonomy, within a digital ecosystem. For this purpose, we distinguish transactions from services (and service providers) by considering Virtual Private Transaction Networks (VPTNs) and Virtual Service Networks (VSNs). These two virtual levels are optimised individually and in respect to each other. The effect of one on the other, can supply us with stability, failure resistance and small-world characteristics on one hand and durability, consistency and sustainability on the other hand. The proposed network design has a dynamic topology that adapts itself to changes in business models and availability of SMEs, and reflects the highly dynamic nature of a digital ecosystem.
\end{abstract}

Index Terms-Digital Ecosystem, Virtual Private Transaction Network, Virtual Service Network, Scale-free network, Business Transaction.

\section{INTRODUCTION}

A number of different views exist on the development of sustainable digital ecosystems, from that of a collaborative environment for business activities, to software infrastructure for open e-business transactions, to the continuous creation of new business model categories and instants. All these different facets can challenge the current infrastructure of our software world. At the same time, the telecoms industry is moving towards the Next Generation Networks (NGNs), and this comes with yet another view of services and applications; the so-called Next Generation Services (NGSs) [2]. Our approach is trying to leverage these developments in creating a business environment which supports dynamic contexts for distributed long-lived transactions in open communities of small-to-medium enterprises (SMEs), proposed in [3],[4].

Current models which provide self-management capabilities at the service level [5], [6], [7], [8] and Quality of Service (QoS) at the virtualization levels [2] can be seen to satisfy the primary requirements of such a "Digital Ecosystem" (DE) environment. However, there is little evidence that they can insulate the collaborative business activities for long-lived transactions from failure - one of the most important requirements for a $\mathrm{DE}$ for business - in the face of the highly dynamic business models of SMEs which cannot be expected to provide the necessary permanent platforms for a connected network.
As a result, keeping the necessary information for coordinating long-lived transactions [3] can be rather challenging, but also the probability of fragmentation in the network cannot be averted. This can have severe consequences in a business environment, since fragmentation in the network directly affects the number of failed transactions [1].

In this paper we propose a model that satisfies some basic requirements of a business network for SMEs in the context of digital ecosystems. We outline the main characteristics of such a business network in Section II. The threat of fragmentation is discussed in Section III along with unsatisfactory conventional approaches to avoid it. In Section IV we introduce a new measurement for categorising platforms. This leads to a P2P network design based on virtual super peers instead of a traditional super peer architecture, a distinction we explain in Section V. Section VI presents early ideas on the changing topology of the network design, and we conclude with ideas for future work in Section VII.

\section{BUSINESS NETWORK PROPERTIES}

The purpose of a business network is to enable networked organisations to engage in distributed business transactions [4] that realise their core business activities. If such a network is to support B2B interactions between SMEs it should be fully distributed (no central point of control for transaction or network operations) and should also offer a consistent model for performing transactions. This means it should be highly resistant to fragmentation - a situation where the network gets divided into smaller isolated networks - as this may prohibit certain collaborative business activities. In addition, there is always the possibility of failure at the transaction level, which should be recoverable and such a procedure must be supported and assisted by the underlying network. The ability for choosing alternative paths/scenarios of execution is another important issue in business transactions.

Meanwhile providing a level of virtualization for applying DE conceptual foundations is necessary. One of the important characteristics of Digital Ecosystems is dynamicity. By their very nature, SMEs are versatile and their business model needs to be refined or adapted on a regular basis. This can transform the business domain or the nature of a business over time. Furthermore, the direct regional effect on business activity time-tables (and availability of SMEs) is another property that needs to be taken into account in a 
digital ecosystem. These are some of the factors that make it necessary for the business network topology of such an environment to be able to adapt itself dynamically (dynamic topology). It should also be noted that loose-coupling is another important characteristic of DE, as SMEs need to preserve their local autonomy, which poses further challenges in covering the above requirements.

For this reason the virtualization in our model is slightly different from that found in the general proposed models (such as [2], [5], [6], [7], [8]). The business activities are at the top level of the model; business activities in the term of transactions which should be distributed (if we are to preserve the local autonomy of SMEs) and recoverable (selfhealing). In previous work [3], [4], we have introduced a transaction model with such characteristics whose design makes use of the inherent diversity of a digital ecosystem. The result of the interaction between participants of a transaction, which comes down to the composition of the corresponding services, provides a virtual connection which is useful for the design of the underlying network. This virtual network, shown as the first conceptual level of virtualisation in Figure 1, is private between transaction participants, and hence the term Virtual Private Transaction Network (VPTN).

The second conceptual level of virtualisation is concerned with links between SMEs (service providers and actual participants of business activities) which provides the structural materials for a business activity (transaction). These structural materials are 'services'. We call such a virtual network a Virtual Service Network (VSN). Figure 1, shows the virtualisation levels of the DE business networks.

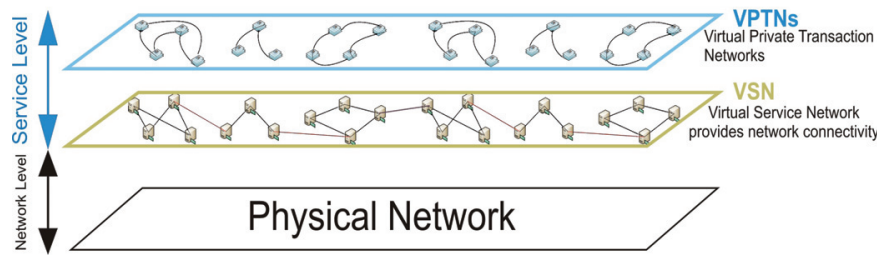

Figure 1. Virtualization in Digital Business Ecosystems

Even though SMEs may be engaging in more than one transaction at a time, a VPTN is typically a fragmented (island) network which connects the participants of a specific business activity. Therefore, nodes in VPTNs are typically in the same business domain (or strongly relevant business domains). For this reason, VPTNs potentially may improve the 'cluster coefficient' of the lower level (VSN) and in exchange VSNs can warranty the diversity for VPTNs (by providing reliable connectivity which makes alternative paths/scenarios feasible for a disconnected VPTN). This may protect business transactions against failure - using alternative paths and scenarios to avoid the costly abortions of long-running transactions is feasible as shown in [4] and is referred to as forward recovery. It can be seen that one of the most important characteristics the VSNs should provide is 'connectivity' - ensuring that there is a network of interconnected nodes.

\section{NETWORK CONNECTIVITY AND FRAGMENTATION}

Connectivity not only is a key point for providing the diversity necessary in a transactional environment, but also can avert unpredicted failures during a fragmentation of the network. That is the reason behind aiming for increased connectivity and the main reason for the cost which most networks pay for avoiding fragmentation. There are several strategies for approaching this problem.

\section{A. Powerful Central Point}

One of the oldest solutions to this problem is to supply a centralised unit for supporting the network connections. This centralised design assumes a powerful central node that manages the whole network and keeps all information about all nodes. However, this solution has the classic problem of a single point of failure, if the central node goes down the whole network collapses, as well as high cost for providing and maintaining the centralised unit. This increases as the number of nodes and associated network traffic increases.

\section{B. Super Peers}

The conventional solution to the problem of fragmentation, which has been used by several P2P networks ([9], [10]) consists of introducing an extra layer to the network, the so-called super peers. Actually the super peers are decentralised servers, which are intended to provide reasonable connectivity and avoid the fragmentation in the network. Depending on the size of the network, the protocol used and the number of super peers, each super peer manages a number of nodes and can check their availability etc. At the same time, each super peer provides a strong link to the other super peers and in this way the design ensures that there is low probability for fragmentation.

\section{Incompatibility with $D E$}

The primary necessity for having super peers is providing stable nodes which are online all of the time. This means super peers are expensive nodes with costly maintenance requirements. It should also be noted that the resources are used for facilitating network operation management tasks. When considering such a solution for a digital ecosystem environment involving SMEs, the question arises as to who is going to provide such nodes? Apart from feasibility issues, most SMEs business models militate against this.

Additionally, during peak time the pressure of high traffic can result in a bottleneck on super peer nodes and because of the connectivity role of super peers, the whole VSNs and consequently VPTNs (and the corresponding business activities of SMEs) will come under serious risk. It could be argued that the problem may be countered by providing maximum facilities and additional resources on super peers, but this may address the problem only temporarily. Powerful super peers will still need to be online and 
monitor the whole network at all times, processing redundant data and producing overheads waste at off-peak times of the network while they will be continuously under pressure at peak time while the network grows (more nodes join).

Moreover, and even if it were possible to find suitable SMEs willing to provide permanent nodes as super peers, these may change their business model and after some time may not find it useful to provide a permanent (and expensive) node anymore. It is not advisable and may not even be possible to force small-to-medium enterprises to be constrained into a static business model and stable behaviour for the sake of stability of the DE infrastructure.

Perhaps even more importantly, the super peers solution results in a static topology for the network as these nodes are pre-selected and their role is pre-determined in the network. This is by no means satisfactory in a highly dynamic environment of a digital ecosystem where the idea is that the network topology changes continuously to adapt to its very usage and demands of the participating entities. The evolving nature of the DE is intended to reflect the congestion of network packages and nodes that change from time to time.

It transpires that dealing with change and adapting to ever changing requirements is unavoidable in the context of a digital business ecosystem. This leads to thinking about a design solution that provides a dynamic topology that continuously evolves to echo changes in the participating entities or nodes. Our approach to the business network design is based on clusters of nodes for providing permanent clusters, rather than permanent nodes as is the case with super peers, and is described in the following sections.

\section{A Measurment for Platform stability}

Since we are dealing with connectivity as a means of avoiding fragmentation of the network, we need a measurement for node stability. It would be unreasonable (and not feasible) to expect nodes to be online all the time and thus stability is determined on the basis of declared availability.

For finding a more precise and computable measurement for node stability, first we introduce an important property for each node, called Expected Availability Time (EAT). The Expected Availability Time is the time when the node is expected to be available and online in the network (Figure 2 shows an example of EAT for a node in the network). The node stability is then calculated as the actual availability of the node against this expected time. These are typically different, since during its EAT the node may be disconnected. These disconnections will reduce stability (reliability) of the corresponding node in the final selection. This notion of stability can be simply calculated as below:
It can be seen that NodeStabil ity $\leq 1$ and the closer NodeStability gets to 1 for a node, the more stable the node is (which can be understood as more reliable or predictable). For calculating the stability function of a node, in the first instance we use its participants in a transaction (other nodes in the same VPTN) to check its availability behaviour (in fact, this can be calculated by the neighbouring nodes in the VSNs too). At the moment, we have considered EAT as a part of SMEs business model which is given by each SME on joining the network. Hence, this is fixed or can only change on the account of the SME providing it. It should be noted that other approaches can be considered for calculating the EAT - for example, it is possible to use an algorithm based on the network neighbourhoods for calculating EAT which would allow it to vary over time.

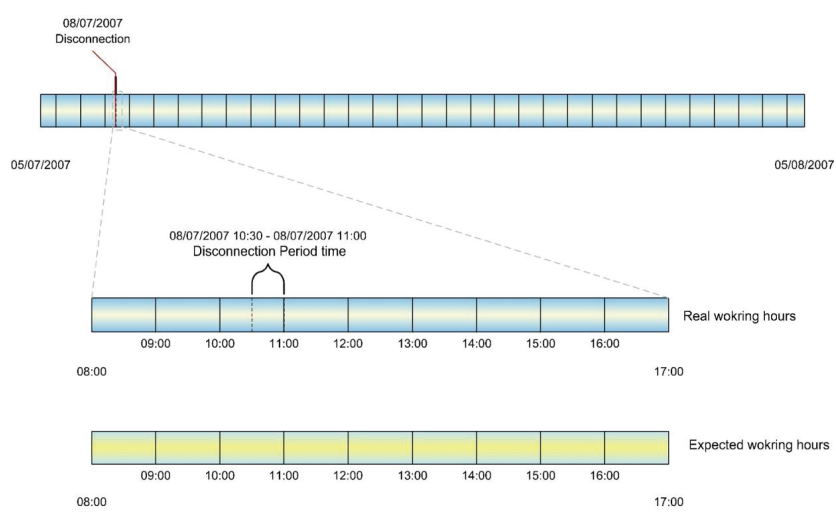

Figure 2. Expected Availability Time

\section{Permanent Clusters \& Virtual Supper Peers}

As mentioned before, in contrast with conventional super peers, we try in our network design to move towards a more dynamic architecture which does not rely on just a few permanent nodes. Central to our approach is finding permanent clusters on the network. More specifically, we are identifying aggregations of stable nodes, where node stability is determined as in the previous section. For doing so, the most stable nodes from different time zones must be chosen, in a way that they cover 24 hours. In fact, we are trying to find permanent clusters through the most stable nodes.

The important part in determining permanent clusters is discovering different aggregations of these time zones which can cover 24 hour availability. Any union of the stable nodes in the aggregations (which provides 24 hour availability coverage) are actual permanent clusters. Figure 3 , shows the simple situation in which the most stable nodes have been selected from two sets of time zones which can cover 24 hour service availability to form permanent clusters.

$$
\text { NodeStability }=\frac{\text { EAT }- \text { DisconnetionPeriods }}{E A T}
$$




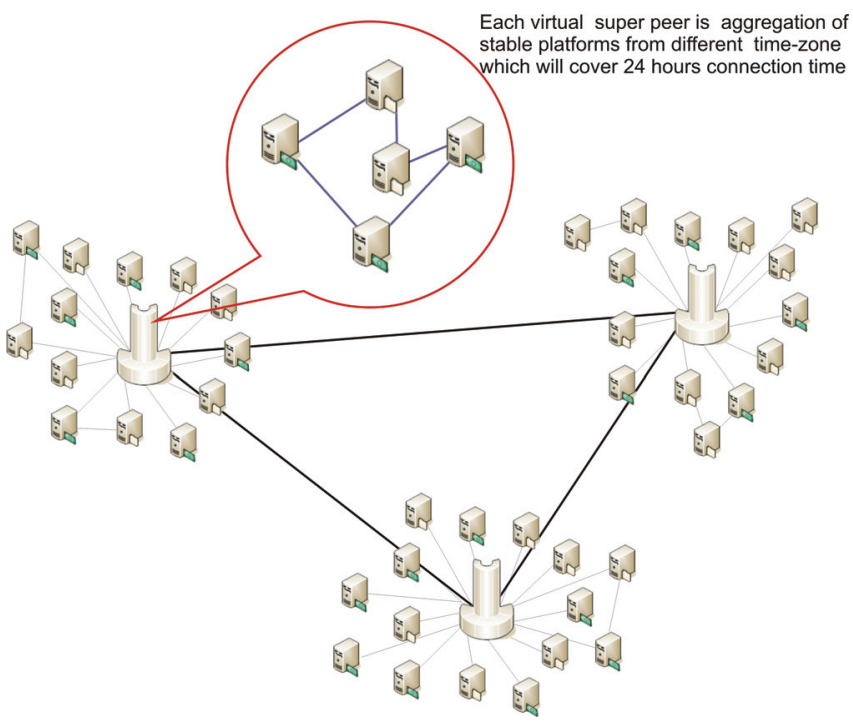

Figure 3. Permanent Clusters and Virtual Super Peers on digital ecosystem

\section{A. Virtual Super Peers}

By using stable nodes from permanent clusters, as is shown in Figure 3, we can create Virtual Super Peers (VSPs) which are effectively permanent clusters of nodes in the network. These can provide the desired stability for the network. The strong connection between the virtual super peers themselves on one hand and the connection between them and their nodes decrease the probability for fragmentation. Depending on the level of reliability required for the network, it is possible to include further redundant stable platforms from each available time zone. For example, in Figure 3 we have included two stable nodes from one timezone and three stable nodes from the other one (the green and creamy signs show different time zones).

In this manner, the good connectivity can cause more reliable transactions at the VPTNs level. Meanwhile the traffic is spread over the virtual super peers and there is less risk of bottleneck at peak time. Nodes within a virtual super peer need to keep information only about nodes in their cluster and about neighbouring VSPs so at off-peak time the amount of redundant information processing is reduced dramatically as compared to the classical super peers solution.

Since choosing stable nodes is a dynamic process (it is done based on the stability function, EAT to Disconnection period of a node during EAT, whose value varies over time) the virtual super peers are also formed dynamically. This means the topology can change from time to time and new nodes can be added to the permanent clusters as the structure of virtual super peers changes. A node can become part of a virtual super peer, when its node stability increases and overcomes some threshold, and nodes that are super peers may not be able to cope with the increased number of connections they get, and possibly increased number of transactions they perform and lose their virtual peer status. Within a digital ecosystem for business, SMEs would be expected to invest at that time (in hardware, processing power, bandwidth etc.) and become again part of a virtual super peer in future. It is in this sense that the topology evolves to reflect the usage and demands of the participants who benefit from and contribute to the 'sustainability' of the network.

Additionally, network congestion can change the maximum level of node stability (Section IV) which in turn affects the selection of the most stable nodes in forming the permanent clusters. High congestion of packages can increase or decrease network reliability (higher traffic on few virtual super peers can potentially create a bottleneck and even cause fragmentation). In a digital business ecosystem, the best part of the traffic is the result of business activities which are effectively long-lived transactions. These have been virtualised in VPTNs and therefore, using the effect of VPTNs for making VSPs and their client nodes, can increase stability of each virtual super peer.

Furthermore, we expect a reasonable cluster coefficient on the account of having VPTN as the main building block which we have seen is formed from a transaction. This means its nodes are in relevant domains - by connecting them to several VSPs we actually increase the probability for that. We also expect a fair distribution degree on the account of propagating links to VSPs. This means that instead of being concerned with individual links for each node, aggregate links of VSPs come into play.

Finally, reusing business activity results (or service-onfly as result of composite services [11]) and explorative service composition [12] are other factors which can be considered for higher performance within a digital business ecosystem and can provide potential for creating so-called virtual vendors.

\section{B. A dynamic algorithm for choosing nodes for VSPS}

In the first step, the most stable node in each VPTN (participants of a transaction) should be selected for keeping vital information about the transaction and its participants. In this sense, the network provide a level of durability without any extra cost from participants and it covers omitted results, a problem relating to preserving as much progressto-date as possible in the event of aborting a transaction. An extended lock mechanism for recovery management in long-lived business transactions has been described in [13] which contains further details on omitted results as well as other aspects of compensation in long-running transactions.

The best candidates for connecting VPTNs together are the most stable nodes in each VPTN. Figure 4 shows the internal structure of each VPTN and the connection between VPTNs. The internal structure of VPTN contains a lot of information from the transaction level such as log structures, lock schemes for ensuring consistency in recovery mentioned above, local coordinator design, formal analysis of the required interactions and compensations, along with alternative scenarios for forward recovery. 


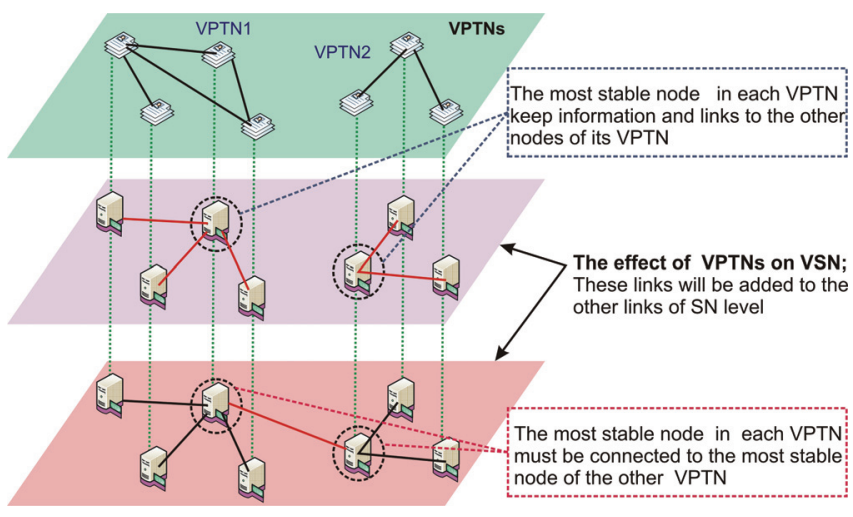

Figure 4. Optimisation from VPTNs to VSN

The direct effect of connecting VPTNs together is rising the cluster coefficient of the network. Conversely, connecting the most stable nodes of VPTNs together provides the opportunity of choosing the best candidate locally between these stable nodes for the permanent cluster. Choosing nodes of the permanent cluster in this way, results in a virtual super peer that provides fair traffic distribution at the VSN level (each virtual super peer will take care of its local VPTNs). The main concept behind forming permanent clusters stays the same, i.e., selecting the most stable nodes from different time zones which can cover 24 hours online time.

\section{The Digital Business Ecosystem Network IN Practice}

As the most stable node in each VPTN is the best candidate for keeping the transaction information, the corresponding business activities will have increased levels of reliability. The fact VPTNs are used initially in the design of the business network, and are connected through their most stable nodes which are determined dynamically, allows in most cases the candidate platform to avoid the full rollback or compensation of the transaction when some participants of the long-lived transaction get disconnected within its duration. This can be considered directly in the design of the recovery mechanism for such a transaction model, as done for example in [13] for the distributed transaction model described in [3]. Another expectation of the network design we have proposed is that the dynamic topology resulting from the selection of virtual super peers, which relies on the stability measurement of each individual platform in each VPTN, reduces the probability of fragmentation. Certain evolutionary models studied in biology exhibit some characteristics of this network design. Meanwhile some practical simulations can compare the theoretical behaviour and practical status of the network in different situations. These aspects are discussed in further detail next.

\section{A. The Network Characteristics}

We have seen that we are dealing with a highly dynamic environment where there is no central point of control and a high probability for failure (in a transaction or the network itself). In the design of a business network for this environment we have considered a dynamic, ever changing to- pology. It would however be desirable to be able to somehow guide the way this topology evolves.

Considering the requirements of DE for business, we propose to draw upon the evolutionary growth of metabolic (signal transduction) networks, as studied in the work of Rzhetsky and Gomez (e.g. [14], [15]) in designing the birth and growth model for an autopoietic P2P network to support long-running business transactions in the OPAALS project (see [1], pp. 77-94). It turns out that the evolutionary growth in molecular networks exhibits scale-free characteristics while it also has some interesting properties with respect to network connectivity. More specifically, the frequency of vertices connected to exactly $\mathrm{k}$ other vertices in metabolic (signal transduction) networks follows a powerlaw distribution. The distribution function degree is equivalent to:

$$
\mathrm{P}(k)=\mathrm{c} \cdot \mathrm{k}^{-\gamma}
$$

where $\mathrm{c}$ is a normalising constant and $\gamma$ diverges across networks (but usually has a value between 1-3; in our simulation was 2.34) and the network follows the classical Barabasi-Albert model [16]. This growth model says that the total number of network vertices is more than three times the number of nodes, which shows the connectivity (even without using VSPs) to be quite good. But there were specific weaknesses which do not seem to be solved without introducing the VSPs conceptual model.

\section{B. The weaknesses of the first model}

The basic network as a scale-free network follows the power law distribution, which means most nodes will have a few links and a few nodes will have a large number of links (see Figure 5). This may result in a high dependency on a few number of nodes which have a large number of links. Such nodes actually play the role of hubs in a typical scale-free network. Thus, the network would already be vulnerable since any smart attack on hubs (or even a series of accidental failures), may cause fragmentation on the network (creating islands in the network), As a consequence, any running business transactions will be discontinued and a fragmented network can be extremely costly to repair (de-fragmentation) as discussed before.

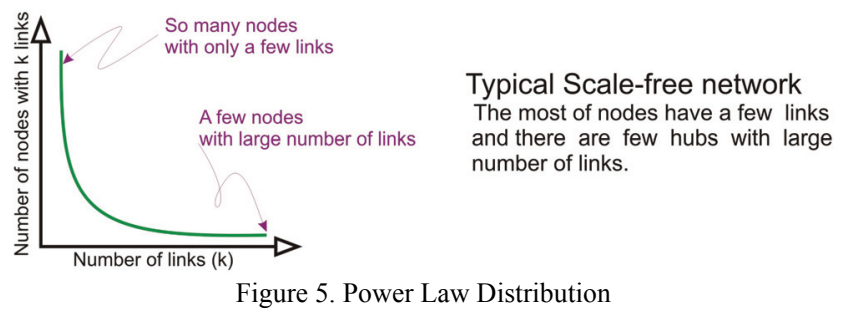

Another problem has to do with the inconsistency of such a model with the dynamicity of a digital ecosystem and the versatility of SMEs business models. Hoping to have stable and permanent hubs to warranty the stability of a few individual nodes is in contrast with the very nature of an ecosystem. Also, SMEs may not provide stable and permanent nodes for hubs at all. Therefore, there is possibility for fragmentation even without any external attack or physi- 
cal failures. As a result of this, the network may suffer regular transition periods between exhibiting the characteristics of a scale-free network and those of a fully random network (with potential islands).

\section{Virtual Super peers' effects}

Our experimentations and results show the proposed model to use the advantages of a conventional scale-free network, but at the same time has built-in capability for coping with events which typical scale-free networks are vulnerable against. This is depicted in Figure 6.

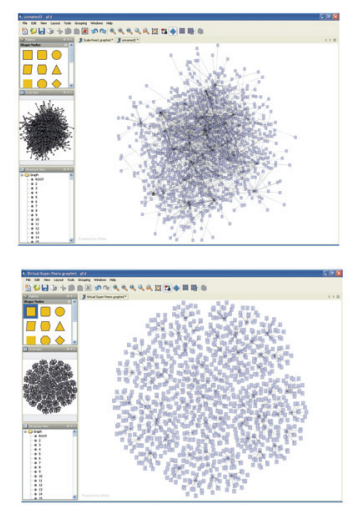

Conventional Scale-free network Power Law Distribution degree Small world properties Vulnerability for smart attacks

Figure 6 Digital Business Ecosystem network in comparison with a conventional network

The major effect of our proposed model is that the actual hubs are virtual super peers. As mentioned in Section V, VSPs are aggregations of several nodes that create a permanent cluster. The dynamic nature of VSPs makes them adaptable to changes in business models or more generally the versatile nature of SMEs. In addition, the fact 'platform stability' varies over time allows for the formation in virtual super peers to also change (if the corresponding platform stability changes).

\section{CONCULUSIONS AND FUTURE WORK}

We have described a P2P design based on the notion of virtual super peers that equips the network with ability for resisting against different types of failure. In contrast with a conventional scale-free network, attacking a few high degree nodes may not destabilise the whole network. This is because in the first step each virtual super peer is made up from several different platforms, from different domains even. At the same time, the dynamic nature of 'permanent clusters' allows for each participant in VSPs to be easily substituted by another stable platform (recall the discussion in Section V).

In addition, platform failures or traffic bottlenecks may not fragment the network or lead it to a transition state between the random graph and its original topology. Actually, even if a node of a permanent cluster fails or experiences high traffic, this will only cause a substitution of the node with another stable node in the closest level. Our actual simulations show the network to follow a fractal model around virtual super peers which can vary depending on the size of the permanent clusters.
The resulting characteristics of this network foster an environment that potentially enhances the ability of SMEs to compete by means of virtual vendors. Through smart composition of services and reuse of profitable (and successful) transaction results can lead to the creation of virtual retailers in place of large enterprise vendors that wish to dominate the infrastructure and rip any potential benefits. Furthermore, the stable network in a collaborative environment can be used in the continuous creation and sharing of knowledge in the form of business models.

\section{Acknowledgements:}

This work was supported by the EU-FP6 funded project OPAALS Contract No 034824

\section{REFERENCES}

[1] A. Razavi, S. Moschoyiannis and P. J. Krause "Preliminary Architecture for Autopoietic P2P Network focusing on Hierarchical SuperPeers, Birth and Growth Models." OPAALS project Deliverable D3.2, 2007 - available at: http://files.opaals.org/OPAALS

[2] Z.B. Daho, Simoni, N. "Towards Dynamic Virtual Private Service Networks: Design and Self-Management", 10th IEEE/IFIP Network Operations and Management Symposium, 2006. NOMS 2006.

[3] A. Razavi, S. Moschoyiannis and P. Krause. "A Coordination Model for Distributed Transactions in Digital Business Ecosystems." In Proc. of IEEE Int'l Conf. on Digital Ecosystems and Technologies (IEEE-DEST 2007), IEEE Computer Society, 2007.

[4] A. Razavi, P. J .Krause and S. K. Moschoyiannis. DBE Project Deliverable D24.5: DBE Distributed Transaction Model, 2006.

[5] B. Martini, F. Baroncelli, P. Castoldi, "A novel service oriented framework for automatically switched transport Network", Symposium on Integrated Network Management, 2005. IM 2005. 2005 9th IFIP/IEEE International. Pub: 15-19 May 2005, pp(s): 295 - 308.

[6] Kristiansen L. et al. "TINA Service Architecture and Specifications", TINA 1.0 DELIVERABLES AND SPECIFICATIONS (available at: http://www.tinac.com/specifications/specifications.htm)

[7] Sahai A. et al. "Automated SLA monitoring for web services", Proceedings of the 13th IFIP/IEEE International Workshop on Distributed Systems: Operations and Management: Management Technologies for E-Commerce and E-Business Applications, p.28-41, October 21-23, 2002.

[8] Z. Li, P.Mohapatra, "QRON: QoS-Aware Routing in Overlay Networks", Service Overlay Networks in the IEEE Journal on Selected Areas in Communications (2004).

[9] B. Y. Beverly, H. Garcia-Molina, " Designing a super-peer network", Proceedings. 19th International Conference on Data Engineering, 2003. Pub: 5-8 March 2003, pp: 49-60

[10] B. Yang, H. Garcia-Molina, "Improving search in peer-to-peer networks", Proceedings. 22nd International Distributed Computing Systems, 2002. pp: 5- 14

[11] Yang, Jian; Papazoglou, M P. and Heuvel, W-J van den (2002), "Tackling the Challenges of Service Composition in EMarketplaces", Twelfth International Workshop on Research Issues in Data Engineering: Engineering E-Commerce/E-Business Systems, 2002. RIDE-2EC 2002 (IEEE Computer society), pp:125-133

[12] Papazoglou M. P., Traverso P., Dustdar S., Leymann F. and Kramer B. J. Service-Oriented Computing Research Roadmap. In Dagstuhl Seminar Proceedings 05462, Service-Oriented Computing (SOC), pp. 1-29, 2006.

[13] A. Razavi, S. Moschoyiannis and P. Krause. Concurrency Control and Recovery Management for Open e-Business Transactions. In Proc. of Communicating Process Architectures (CPA 2007), 2007.

[14] A. Rzhetsky, S. Gomez, "Birth of scale-free molecular networks and the number of distinct DNA and protein domains per genome", Bioinformatics, 17(10):988-996, 2001.

[15] G. Karev, Y. Wolf, A. Rzhetsky, F. Berezovskaya, E. Koonin "Birth and death of protein domains: A simple model of evolution explains power law behavior", BMC Evolutionary Biology, 2:18, 2002

[16] A. L Barabasi, R. Albert "Emergence of Scaling in Random Networks". Science 286(5439): 509-512, 1999 